ARTICLE

DOI: $10.1038 / \mathrm{s} 41467-018-06342-7$

\title{
Multibandgap quantum dot ensembles for solar-matched infrared energy harvesting
}

Bin Sun (10 1, Olivier Ouellette (10 1, F. Pelayo García de Arquer ${ }^{1}$, Oleksandr Voznyy (10 1, Younghoon Kim ${ }^{1,5}$, Mingyang Wei ${ }^{1}$, Andrew H. Proppe (1,2,3, Makhsud I. Saidaminov ${ }^{1}$, Jixian Xu' ${ }^{1}$, Mengxia Liu', Peicheng $\mathrm{Li}^{4}$, James Z. Fan', Jea Woong Jo1, Hairen Tan (10 1, Furui Tan'1, Sjoerd Hoogland', Zheng Hong Lu (i) ${ }^{4}$, Shana O. Kelley (i) ${ }^{2,3} \&$ Edward H. Sargent (iD ${ }^{1}$

As crystalline silicon solar cells approach in efficiency their theoretical limit, strategies are being developed to achieve efficient infrared energy harvesting to augment silicon using solar photons from beyond its $1100 \mathrm{~nm}$ absorption edge. Herein we report a strategy that uses multi-bandgap lead sulfide colloidal quantum dot (CQD) ensembles to maximize short-circuit current and open-circuit voltage simultaneously. We engineer the density of states to achieve simultaneously a large quasi-Fermi level splitting and a tailored optical response that matches the infrared solar spectrum. We shape the density of states by selectively introducing largerbandgap CQDs within a smaller-bandgap CQD population, achieving a $40 \mathrm{meV}$ increase in open-circuit voltage. The near-unity internal quantum efficiency in the optimized multibandgap CQD ensemble yielded a maximized photocurrent of $3.7 \pm 0.2 \mathrm{~mA} \mathrm{~cm}^{-2}$. This provides a record for silicon-filtered power conversion efficiency equal to one power point, a $25 \%$ (relative) improvement compared to the best previously-reported results.

\footnotetext{
${ }^{1}$ Department of Electrical and Computer Engineering, University of Toronto, 10 King's College Road, Toronto, ON M5S 3G4, Canada. ${ }^{2}$ Department of Pharmaceutical Science, Leslie Dan Faculty of Pharmacy, University of Toronto, Toronto, ON M5S 3G4, Canada. ${ }^{3}$ Department of Biochemistry, Faculty of Medicine, University of Toronto, Toronto, ON M5S 3M2, Canada. ${ }^{4}$ Department of Material Science and Engineering, University of Toronto, 184 College St, Toronto, ON M5S 3E4, Canada. ${ }^{5}$ Present address: Convergence Research Center for Solar Energy, Daegu Gyeongbuk Institute of Science and Technology, Daegu 42988, Republic of Korea. These authors contributed equally: Bin Sun, Olivier Ouellette, F. Pelayo García de Arquer. Correspondence and requests for materials should be addressed to E.H.S. (email: ted.sargent@utoronto.ca)
} 
P hotovoltaics accounted for $1.3 \%$ of the global energy supply in 2016 , a number that is projected to increase to $20 \%$ by $2050^{1}$. As crystalline silicon (cSi) solar cells approach their theoretical efficiency limit ${ }^{2}$, complementary strategies that further improve efficiency - without introducing significant additional cost - provide avenues to lower further the price of solar electricity.

With an indirect bandgap of $1.1 \mathrm{eV}$ corresponding to an absorption edge at $1100 \mathrm{~nm}$, Si solar cells leave up to $20 \%$ of the solar power reaching the Earth's surface unabsorbed. Efficient infrared energy harvesting that could complement $\mathrm{Si}$ absorption is a promising route to achieve broadband solar energy conversion, which is predicted to offer up to $6 \%$ additional power points on top of existing cSi photovoltaic solutions ${ }^{3,4}$.

Colloidal quantum dots (CQDs) combine facile and broad spectral tunability via quantum-size tuning ${ }^{5,6}$ with inexpensive manufacturing arising from their solution-processing. In the last decade, intensive efforts have focused on improving CQD synthesis, surface passivation, film formation, and device engineering; and these have led to great strides in increasing the performance of CQD photovoltaics ${ }^{6-12}$. IR CQD solar cells, on the other hand, have remained comparatively underexplored, and best IR-filtered PCEs lie below $0.5 \% 4,13,14$.

An acute challenge in CQD solar cells is to realize simultaneously high short-circuit current $\left(J_{\mathrm{SC}}\right)$ and high open-circuit voltage $\left(V_{\mathrm{OC}}\right)$. As the size of QDs is increased and their bandgap shrinks so that more IR photons can be absorbed - a crucial step to harvest the solar power beyond $1100 \mathrm{~nm}-V_{\mathrm{OC}}$ decreases due to the smaller bandgap and the presence of energy losses $\left(E_{\text {loss }}\right)$. $E_{\text {loss }}$ is defined as the deficit in $V_{\mathrm{OC}}$ compared to the detailed balance limit for $V_{\mathrm{OC}}$ at a given bandgap ${ }^{15,16}$, and in CQD photovoltaics it stems primarily from bandtail states and recombination at defects. While energy losses on the order of $0.1-0.2 \mathrm{eV}$ are observed for highly crystalline and low-defect materials such as cSi, CQDs are characterized by significantly higher values, reaching $0.4 \mathrm{eV}^{8,17,18}$. The reduction of bandtail states to decrease this detrimental loss has therefore been a widespread theme in recent work ${ }^{9}$. The absorption/extraction compromise, which limits the thickness of the CQD active layer to a few hundreds of nanometers, represents an additional impediment to harvesting fully the infrared portion of the solar spectrum $^{19}$.

In this work, we revisit the conditions under which $V_{\mathrm{OC}}$ is pinned in CQD ensembles. In doing so, we find a regime wherein $V_{\mathrm{OC}}$ - rather than being rapidly pinned by the lowest bandgap component in a quantum dot ensemble ${ }^{20}$ - is instead related linearly to the bandgap of the ensemble constituents. In this regime, the $V_{\text {OC }}$ for a given bandgap can be increased by the judicious addition of a larger bandgap species that modifies the density of states. We exploit this phenomenon and design CQD multi-bandgap ensembles that, by virtue of a tailored density of states and by spectrally matching the IR solar spectrum, simultaneously attain for the first time high $V_{\mathrm{OC}}$ and high $J_{\mathrm{SC}}$ of $0.4 \mathrm{~V}$ and $3.7 \pm 0.2 \mathrm{~mA} \mathrm{~cm}^{-2}$, respectively, more than $30 \%$ higher than previously reported values for both parameters ${ }^{14}$. As a result, we achieve cSi-filtered PCE of $1 \%$ - a record in infrared CQD PV ${ }^{4,14}$.

\section{Results}

$V_{\text {OC }}$ modulation in multi-bandgap quantum dot ensembles. Under illumination, the electron quasi-Fermi level increase in solar cells made from a single population of CQDs is dictated by the excited carrier density that can be sustained in the conduction band in steady state ${ }^{8}$. The overlap of the Fermi-Dirac occupation function at the quasi-Fermi level $f\left(E, E_{\mathrm{QFL}}\right)$ and the density of states (DOS) at the CQD conduction $g_{\mathrm{CB}}(\mathrm{E})$ band determines this photoexcited electron density (Fig. 1a):

$$
\Delta n=\int_{E_{\mathrm{C}}}^{\infty} f\left(E, E_{\mathrm{QFL}}\right) g_{\mathrm{CB}}(E) \mathrm{d} E
$$

A similar expression holds for photoexcited holes in the valence band. Mixing different CQD ensembles can be used to modify proportionately the effective DOS, which affects the overlap with the Femi-Dirac distribution of electrons depending on the relative weight of the populations and the difference in energy $\Delta E$ of the mixed dot ensembles (Fig. 1b). For a given photoexcited charge density $\Delta n, E_{\mathrm{QFL}}$ will therefore increase if the relative density of lower energy states is reduced. We note that the F-D distribution is appropriate to describe the occupation

C

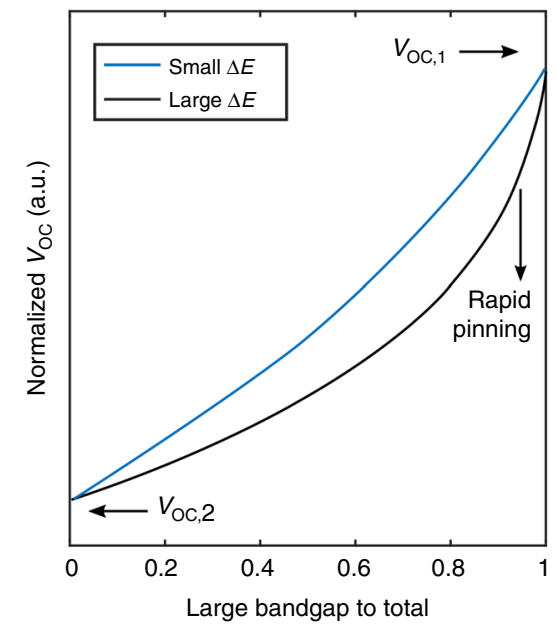

Fig. 1 Open-circuit modulation in multi-bandgap QD ensembles under illumination. a A single population of small bandgap CQDs; b overlap of the FermiDirac occupation function at the quasi-Fermi level $f\left(E_{,} E_{\mathrm{QFL}}\right)$ and the density of states (DOS) at the CQD conduction $g_{\mathrm{CB}}(E)$ band; $\mathbf{c} V_{\mathrm{OC}}$ behavior upon $C Q D$ mixing depending on the energy offset of the large bandgap inclusions in the mixed CQD films 
probability not only in bands, but also of discrete energy states such as, for example, in the monoatomic ideal gas ${ }^{21}$, and, more broadly, in systems with single-particle energy levels ${ }^{22}$. FermiDirac statistics apply only if the particles in the system can reach thermal equilibrium. Using ultrafast transient absorption spectroscopy (Supplementary Fig. 7 and 8), we verified experimentally that, in the pure and mixed CQD mixes, photoexcited electrons and holes ${ }^{23}$ thermalize to the nearby available states in a few nanoseconds, well before they are lost to recombination, and thus do reach thermal equilibrium within their band.

To quantify this effect, we employed a band-filling model ${ }^{24,25}$ and calculated the impact of CQD size mixing on $V_{\mathrm{OC}}$. The conduction and valence DOS were built assuming Gaussian CQD size distributions and using the following size-to-bandgap relation: ${ }^{26}$

$$
E_{\mathrm{G}}=\frac{1}{0.0252 x^{2}+0.283 x}
$$

where $E_{\mathrm{G}}$ is the bandgap $(\mathrm{eV})$ and $x$, the quantum dot diameter $(\mathrm{nm})$. To retrieve the quasi-Fermi level splitting, which corresponds to the upper $V_{\mathrm{OC}}$ limit, the steady state photoexcited charge generation rate is set equal to the recombination rate, which is assumed to be dominated by mid-gap tail states ${ }^{27}$. Details and calculation parameters are given in Supplementary Note 1 and Supplementary Fig. 1 and 2.

Different regimes are identified in the $V_{\mathrm{OC}}$ behavior upon CQD mixing (Fig. 1c) as a function of the energy offset. When $\Delta E$ is large compared to the FWHM of the DOS (given by the size distribution), the open-circuit voltage is rapidly pinned to the $V_{\mathrm{OC}}$ of the smallest-bandgap population. This case represents the conventional scenario in which, in a CQD film, the presence of narrow bandgap outliers and deep tail states dramatically reduces $V_{\mathrm{OC}}$. As $\Delta E$ diminishes and the broadened DOS overlaps progressively more with $f(E)$, the open-circuit voltage shows an almost linear dependence on the $V_{\mathrm{OC}}$ corresponding to the individual populations of the CQD ensemble. We therefore predict that modifying the DOS by mixing in CQDs with a slightly higher bandgap should have an appreciable beneficial effect on $V_{\mathrm{OC}}$.

Transport characteristics of multi-bandgap CQD ensembles. We then proceeded to make films of CQD ensembles based on a solution-phase exchange method to replace the as-synthesized oleic acid capped CQDs with short inorganic halide ligands. Our solution exchange is based on a previously-reported protocol ${ }^{28}$ for $1150 \mathrm{~nm}$ (large bandgap, L) and $1250 \mathrm{~nm}$ (small bandgap, S) CQDs. We optimized the solution exchange protocol as follows: ${ }^{14,28}$ for $1150 \mathrm{~nm}$ CQDs, we kept $\mathrm{PbI}_{2}$ and $\mathrm{Pb}(\mathrm{SCN})_{2}$ at the same concentration as our previous work and modified the concentration of ammonium acetate (AA) from $10 \mathrm{mM}$ to $60 \mathrm{mM}$ in dimethylformamide (DMF) (Supplementary Fig. 3). When we increase the AA concentration, $V_{\mathrm{OC}}$ decreases while FF and PCE increase before decreasing as well, which is ascribed to surface passivation and change in residual $\mathrm{OA}$ on the surface. The optimal concentration of AA of $20 \mathrm{mM}$ was found for the 1150 $\mathrm{nm}$ CQD ligand exchange. We also optimized the $1250 \mathrm{~nm}$ CQD ligand exchange (Supplementary Fig. 4) by adjusting the AA concentration and added butylamine (BTA) to assist ligand exchange. In this case, the optimal concentration was found experimentally to be $60 \mathrm{mM}$ for AA and $40 \mathrm{mM}$ for BTA. We additionally performed X-ray photoelectron spectroscopy (XPS) to study the surface passivation (Supplementary Fig. 4c). The addition of BTA allows for more organics (oleic acid) and iodide ions to remain on the CQD surface, as indicated by the higher ratio of I:S and C:S compared to the control ligand exchange without $\mathrm{BTA}^{17,29}$. We finally mixed the individual solutions (with the choice of ratio explored throughout this work) prior to CQD film formation.

To characterize the charge mobility and density of tail states for different quantum dot ensembles, we carried out field-effect transistor (FET) measurements (Fig. 2) ${ }^{30}$. We employed a bottom-gate top-contact configuration (Fig. 2a). The FET transfer characteristics for all the studied mixtures reveal the characteristic $n$-type character of halide-treated CQD films (Fig. 2b).

We retrieved the density of in-gap states from the measured transfer characteristics. By analyzing the exponential increase of the drain current below $V_{\mathrm{TH}}$, which corresponds to transport through in-gap states, we obtain the density of in-gap states. The tail state distribution is calculated using the following equation: ${ }^{28}$

$$
N_{\mathrm{td}}=\left[\left(\frac{S \cdot e}{k T \cdot \ln (10)}-1\right) \cdot \frac{C_{\mathrm{i}}}{e}\right]^{2} \cdot \epsilon_{0} \epsilon_{\mathrm{r}}^{-1}
$$

where $S$ is the sub-threshold swing, the slope of the gate voltage vs. the log drain current between turn-on voltage and $V_{\mathrm{TH}}$ that defines the boundary between the subthreshold and transport regime; $\epsilon_{0}$ is the vacuum permittivity; $\epsilon_{\mathrm{r}}$ is the electric constant of the film, estimated to be $10.9^{31}$. After integrating the tail state distribution between the subthreshold and transport regime as shown in Fig. 2c for the mixture (weight ratio of 2 to 1), we obtain the density of tail states $\left(N_{\mathrm{T}}\right)$ (Supplementary Fig. 5) plotted in Fig. 2d. The pure large gap CQD film exhibits a $N_{\mathrm{T}}$ of $1.5 \pm 0.2 \times$ $10^{16} \mathrm{~cm}^{-3}$ (Supplementary Fig. 5), which is close to that of solution exchanged $950 \mathrm{~nm}$ PbS CQDs ${ }^{28}$. The pure small-gap CQD film shows a two orders of magnitude lower $N_{\mathrm{T}}$ of $2.6 \pm$ $0.5 \times 10^{14} \mathrm{~cm}^{-3}$ compared to the pure large gap CQD film (Fig. 2d), a finding we ascribe to better surface passivation. We also compared the transport properties of small bandgap dots exchanged with and without the BTA additive (Supplementary Fig. 6). The CQD film exchanged without BTA exhibits a $N_{\mathrm{T}}$ of $5.2 \pm 0.4 \times 10^{16} \mathrm{~cm}^{-3}$, while the addition of BTA lead to a much lower $N_{\mathrm{T}}$ of $2.6 \pm 0.5 \times 10^{14} \mathrm{~cm}^{-3}$, again due to better surface passivation. The CQD mixtures containing 33,50 , and $67 \%$ of large bandgap CQDs exhibit a $N_{\mathrm{T}}$ of $2.8 \pm 0.4 \times 10^{15}, 3.6 \pm 0.3 \times$ $10^{15}$, and $1.7 \pm 0.3 \times 10^{15} \mathrm{~cm}^{-3}$, respectively, an order of magnitude lower than that of the pure large gap CQDs, indicating that the mixtures should have similar or even better carrier transport compared to the large bandgap CQD films.

In addition to obtaining tail density, we also extracted charge carrier mobility from FET measurements (Fig. 2d). The carrier mobility is calculated from the slope of $I_{\mathrm{DS}}$ vs. $V_{\mathrm{GS}}$ according to the equation $I_{\mathrm{DS}}=\mu C_{i} \frac{W}{L}\left(V_{\mathrm{GS}}-V_{\mathrm{TH}}\right) V_{\mathrm{DS}}$, where $\mu$ is the carrier mobility in the linear regime; $I_{\mathrm{DS}}$ is the drain current; $L$ and $W$ are the channel length $(50 \mu \mathrm{m})$ and channel width $(2.5 \mathrm{~mm})$ respectively; and $V_{\mathrm{GS}}$ and $V_{\mathrm{TH}}$ are the gate voltage and threshold voltage, respectively. The pure large-gap CQD film has an electron mobility of $0.052 \pm 0.003 \mathrm{~cm}^{2} \mathrm{~V}^{-1} \mathrm{~s}^{-1}$, while the pure small-gap CQD film shows a lower mobility of $0.020 \pm 0.002$ $\mathrm{cm}^{2} \mathrm{~V}^{-1} \mathrm{~s}^{-1}$, which may be due to the residual oleic acid ligands on the CQD surface. The CQD films with inclusions of large bandgap CQDs of 33, 50, and 67\% exhibit mobilities of $0.026 \pm$ $0.004,0.023 \pm 0.004$, and $0.021 \pm 0.003 \mathrm{~cm}^{2} \mathrm{~V}^{-1} \mathrm{~s}^{-1}$, respectively. In addition, we studied charge carrier transport between the two differently-sized distributions using ultrafast transient absorption spectroscopy (Supplementary Fig. 7 and 8). We found that the wide size dispersity allows for photoexcited charges to be thermally excited into larger and/or smaller dots, thereby thermalizing into the nearby available states in a few nanoseconds. We also conducted ultraviolet photoelectron spectroscopy (UPS) (Supplementary Fig. 9) to determine the position of the 
a
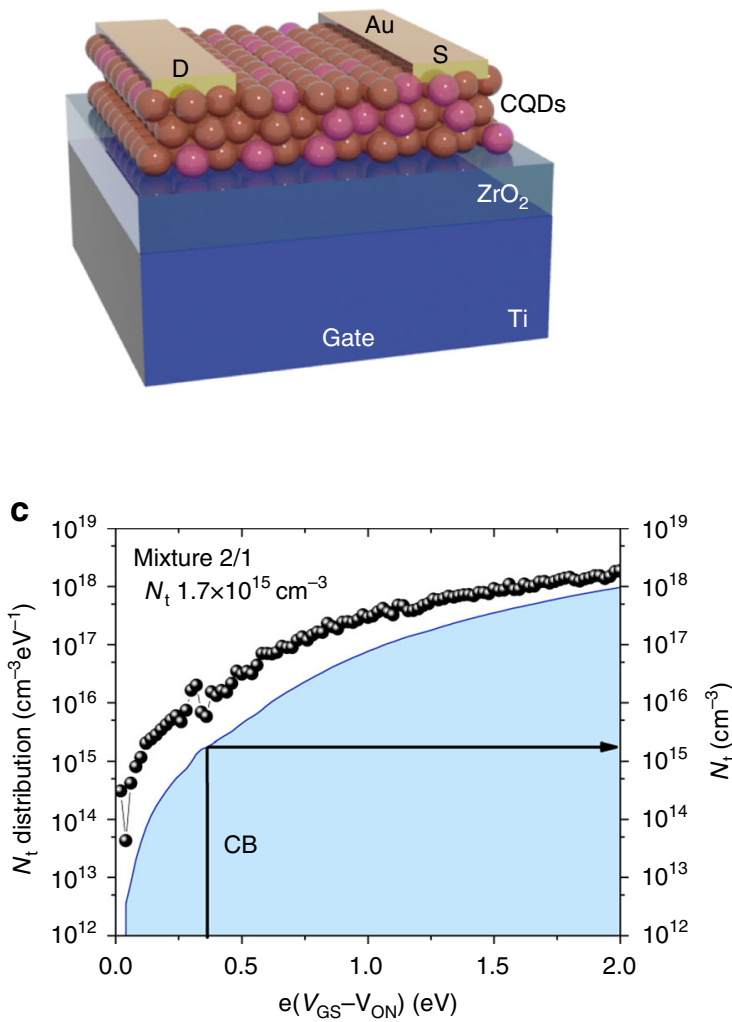

b

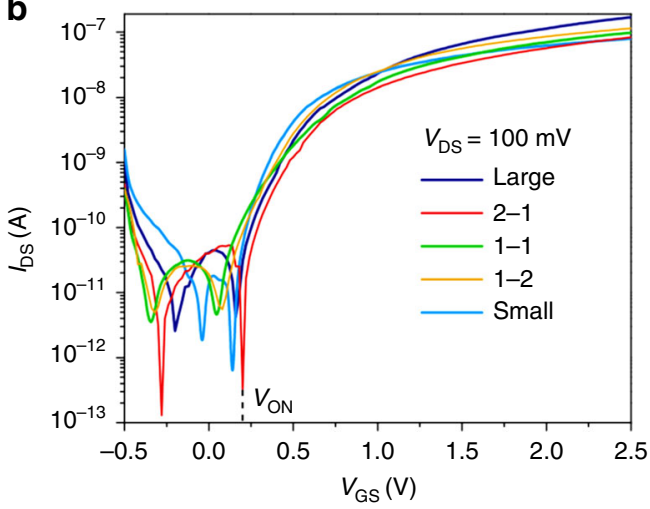

d

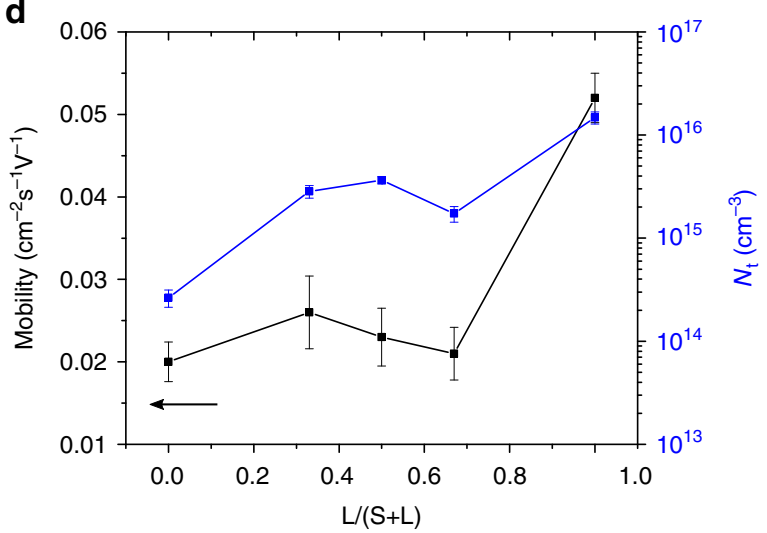

Fig. 2 Transport properties of CQD multi-bandgap ensembles a bottom-gate top-contact field-effect transistor structure; transfer characteristics of pure CQD and multi-bandgap CQD ensembles with different weight ratio of large bandgap $(\mathrm{L})$ to small bandgap $(\mathrm{S})$ CQDs showing onset voltage $\left(V_{\mathrm{ON}}\right)$ b, transfer characteristics of pure and mixed CQDs with different weight ratios of large bandgap (L) to small bandgap (S); c tail state density ( $\left.N_{T}\right)$ of the optimal CQD mixture (weight ratio of 2 to 1 ) as a function of gate bias as calculated with Eq. (3); $\mathbf{d}$ mobility and trap density as a function of the inclusion of $L$ in the mixed films
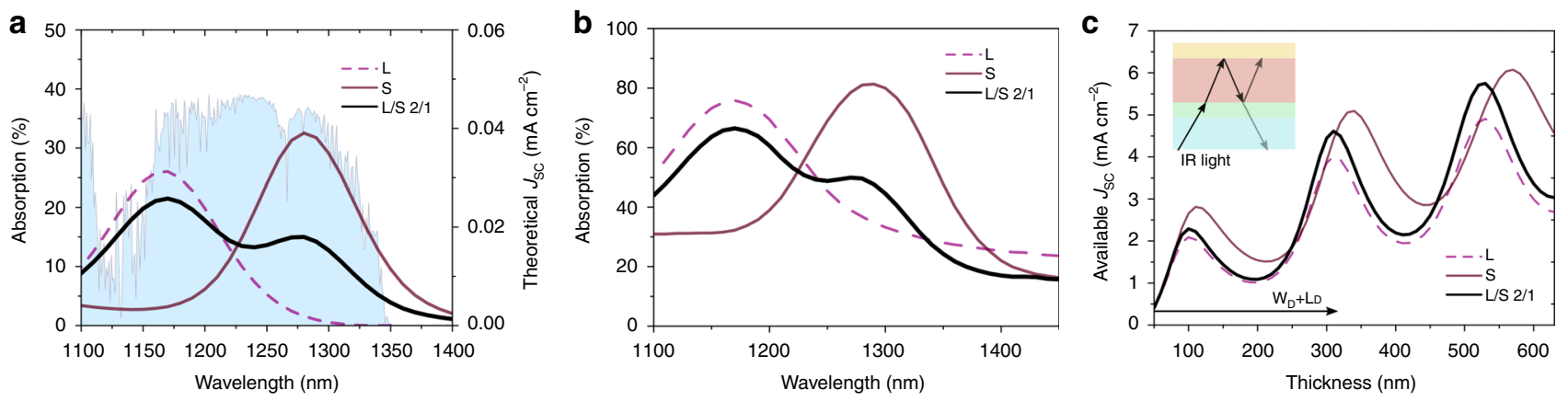

Fig. 3 Expected $J_{S C}$ from multi-bandgap CQD ensembles. absorptance measured from (a) the CQD films on glass and $\mathbf{b}$ complete solar cell devices including the gold electrode mirror; c calculated $J_{S C}$ as a function of CQD film thickness

energy levels of the single size CQDs, and confirmed that they have energy levels needed for band alignment.

Tailoring the multi-bandgap CQD ensembles spectral response. The band-filling model and FET analysis indicate that the mixtures can achieve improved $V_{\mathrm{OC}}$ and comparable charge transport properties. We sought to leverage this property and turned our attention to the optical behavior of the multi-bandgap CQD ensemble and aimed to maximize the overlap of light absorption with the cSi-filtered infrared solar spectrum.

Figure 3a shows the single pass absorptance of CQD films of the same thickness $(300 \pm 10 \mathrm{~nm})$ on optical glasses, where the 2:1 (large bandgap: small gap) films have a lower absorptance maxima than pure CQDs ( $30 \%)$. The mixtures do not show significantly higher IR photon absorption than the pure CQD films. In a complete CQD solar cell, however, the gold backelectrode serves also as a mirror. The resulting reflection contributes to the device absorptance and introduces resonant absorption. This is due to interference between the forwardpropagating light from the illuminated side and the backwardpropagating light reflected on the gold electrode and can be controlled and optimized by adjusting the active layer thickness ${ }^{13,32}$. We thus measured the total absorption through complete PV devices (Fig. 3b). We observed that light absorption 
a

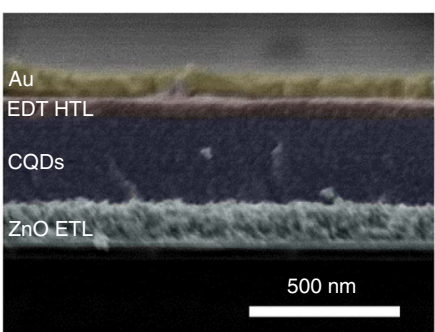

d

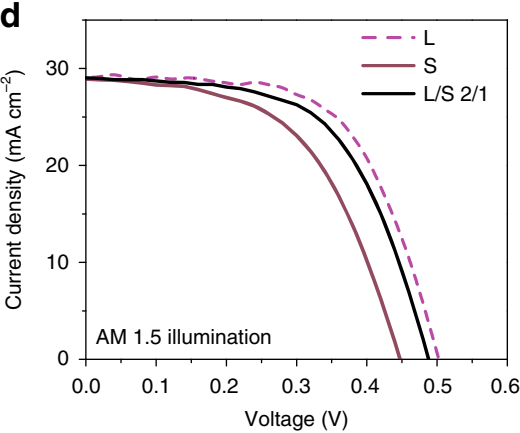

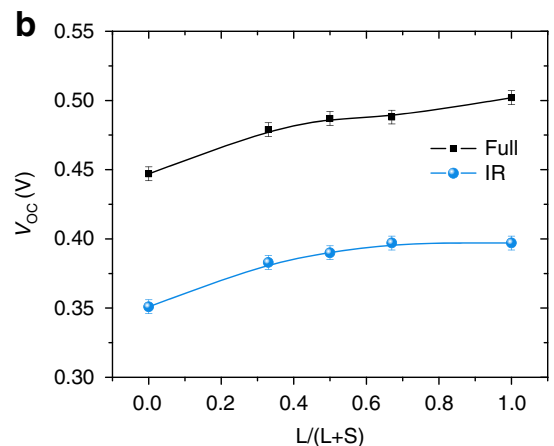

C
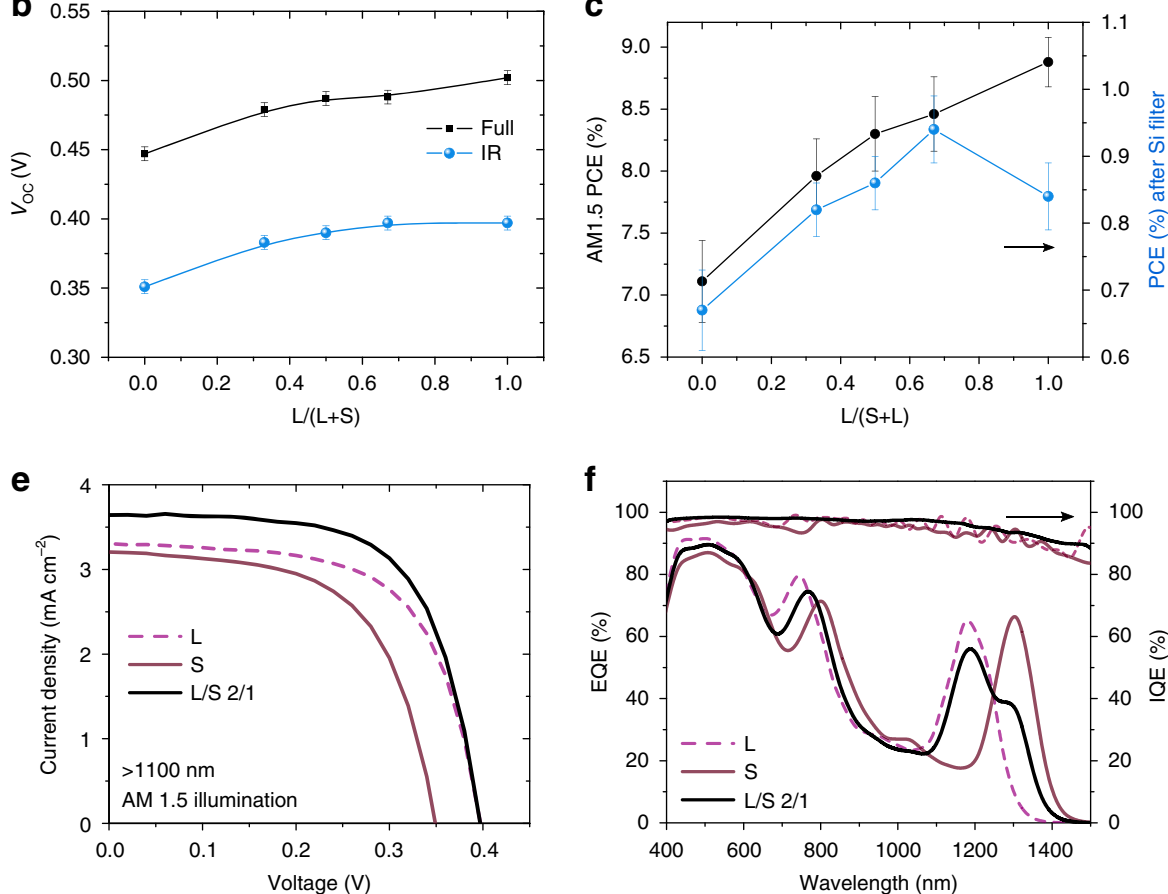

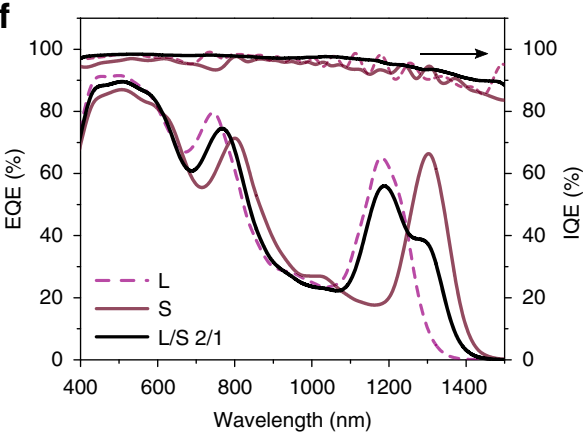

Fig. 4 PV Device architecture and performance. a Device architecture and cross-sectional SEM image of the best mixed CQD film solar cell. Measured (b) $V_{O C}$ and $\mathbf{c}$ PCE with different inclusions of large bandgap CQDs. d J-V characteristics under AM1.5 G, e $J-V$ characteristics after $1100 \mathrm{~nm}$; $\mathbf{f}$ EQE curves and IQE curves of optimal single and mixed CQD solar cell devices

in the mixtures is enhanced at certain wavelengths, which contribute to additional photo-generated current. To confirm the effect of optical resonance, we additionally measured light absorption in CQD films before Au deposition (Supplementary Fig. 10), which lack the resonant absorption peaks present in the absorption spectra of devices containing the Au back mirror, thus confirming the role of the resonant mechanism.

To optimize the total IR absorption, we calculated the available $J_{\mathrm{SC}}$ as the thickness of the active layer varies using the transfermatrix method ${ }^{32,33}$ (Fig. 3c and Supplementary Fig. 11). Pure large bandgap dots and 2 to 1 mixture films have a local $J_{\mathrm{SC}}$ maximum at a thickness of about $300 \mathrm{~nm}$, while the pure small bandgap dots can absorb more light at about $340 \mathrm{~nm}$, which is due to the absorption peak position difference. The available $J_{\mathrm{SC}}$ decreases after the first local maximum, and much thicker CQD films (above $500 \mathrm{~nm}$ ) are required for a net increase in $J_{\mathrm{SC}}$. For such a large thickness, the efficiency of charge carrier extraction will be dramatically reduced, as the diffusion length in these CQD solids is in the order of hundreds of nm. Based on these findings, we narrowed our attention to 2 to 1 mixtures and active layer thicknesses ranging from 200 to $350 \mathrm{~nm}$.

PV device performance. We then characterized the photovoltaic performance of solar cells employing multi-bandgap CQD ensembles (Fig. 4). The devices consist of a $\mathrm{ZnO}$ layer, acting as an electron acceptor; an active layer formed of $\mathrm{PbS}$ CQD ensemble; EDT-exchanged PbS CQDs as the hole acceptor, and thermally evaporated gold as the top electrode.

The open-circuit voltage shows the predicted trend upon quantum dot mixing (Fig. 4b). The AM1.5 $V_{\mathrm{OC}}$ for large bandgap is $0.50 \mathrm{~V}$, and $0.45 \mathrm{~V}$ for small bandgap CQDs. The $V_{\text {OC }}$ of $0.45 \mathrm{~V}$ for small-gap CQDs is higher than previous reports for similar sizes $(0.38 \mathrm{~V})$, which we ascribe to the lower $N_{\mathrm{T}}$ stemming from better passivation. The $V_{\mathrm{OC}}$ of mixtures gradually shifts between the two pure CQDs, relating to the weight inclusions almost linearly as expected from the state-filling model. We calculated the energy loss dependence on the inclusion of large bandgap CQDs in mixed CQD films under AM1.5 irradiation (Supplementary Fig. 12) and found that the mixed CQDs exhibit the lowest $E_{\text {loss }}(<0.27 \mathrm{eV})$, lower than that of the large and small bandgap CQDs (0.33 and $0.30 \mathrm{eV}$, respectively).

We then characterized the PV devices after an $1100 \mathrm{~nm}$ longpass filter to replicate the effect of a silicon front cell. The mixture with $67 \%$ of large bandgap CQDs shows an IR $V_{\text {OC }}$ of $0.40 \mathrm{~V}$, similar to that of pure large bandgap CQDs films. This further demonstrates the benefit of multi-bandgap CQD ensembles to maximize open-circuit voltage. With fewer inclusions of large-gap CQDs, the IR $V_{\mathrm{OC}}$ of the mixtures gradually decreases with the decreased portion of large-gap CQDs. The similar IR $V_{\mathrm{OC}}$ of mixed CQD films compared to pure large bandgap CQD films can be attributed to the lower $N_{\mathrm{T}}$ than that of pure large bandgap CQD films, which reduces trap-assisted recombination, lowering the drop of $V_{\mathrm{OC}}$ with the reduced light intensity. We also investigated the impact of a higher bandgap difference between the mixed CQDs on the resulting $V_{\text {OC }}$ (Supplementary Fig. 13). The $V_{\mathrm{OC}}$ of mixes of CQDs with exciton peaks at $1150 \mathrm{~nm}$ and $1512 \mathrm{~nm}$ is quickly pinned to that of the small bandgap CQDs, in agreement with the theoretical model.

Multibandgap CQD ensembles exhibit a superior IR PCE compared to pure CQD films (Fig. 4c, Supplementary Table 3). The best IR PCE of $0.95 \pm 0.04 \%$ was obtained in the mixture containing $67 \%$ large bandgap CQDs, with a $0.40 \pm 0.01 \mathrm{~V} V_{\mathrm{OC}}, 3.7$ $\pm 0.2 \mathrm{~mA} \mathrm{~cm}^{-2} J_{\mathrm{SC}}$, and a $65 \pm 1 \%$ fill factor (FF). The best largebandgap CQD films, on the other hand, led to a PCE of $0.84 \pm$ $0.03 \%$ with $V_{\mathrm{OC}}, J_{\mathrm{SC}}$, and $\mathrm{FF}$ at $0.40 \pm 0.01 \mathrm{~V}, 3.3 \pm 0.2 \mathrm{~mA} \mathrm{~cm}^{-2}$, $64 \pm 1 \%$; the small bandgap CQD solar cells yielded a PCE of $0.67 \pm$ $0.05 \%$ with $V_{\mathrm{OC}}, J_{\mathrm{SC}}$, and FF at $0.35 \mathrm{~V}, 3.2 \pm 0.2 \mathrm{~mA} \mathrm{~cm}^{-2}, 60 \pm 1 \%$. The device performance under unfiltered AM1.5 G illumination is presented in Supplementary Fig. 18 and Supplementary Table 2 for reference.

We tested three different multi-bandgap CQD ensemble configurations, containing large bandgap CQDs from 33 to 
$67 \%$; all these three compositions showed at least $20 \%$ improvement compared to the small bandgap samples. The enhancement of absorption in mixtures containing $67 \%$ largebandgap CQDs yields an enhanced $J_{\mathrm{SC}}$ of $3.7 \mathrm{~mA} \mathrm{~cm}^{-2}$, calculated from the EQE:

$$
J_{\text {SC }}=q \int_{0}^{\infty} \operatorname{EQE}(\lambda) \gamma_{\mathrm{i}}(\lambda) \mathrm{d} \lambda
$$

where $\gamma_{\mathrm{i}}(\lambda)$ is the incident solar photon flux spectrum. Tailoring the absorption spectrum leads to this increase in $J_{S C}$ by better matching the external quantum efficiency (EQE) spectrum to the solar spectrum over the $1100 \mathrm{~nm}$ to $1400 \mathrm{~nm}$ spectral range (Supplementary Fig. 14). The EQE of the best mixed CQD device is wider than its pure counterparts, as seen by the increase in fullwidth half-maximum (FWHM) of the exciton peak (Supplementary Fig. 15), which in turn leads to an increase in photocurrent when the absorption spectrum is well matched to the solar spectrum. The shape of the exciton peak and its FWHM was tuned to the solar spectrum to increase $J_{\mathrm{SC}}$ while minimizing $V_{\mathrm{OC}}$ loss. We note that the extended FWHM of the exciton peak did not improve $J_{\mathrm{SC}}$ under full-AM1.5-spectrum one-sun conditions (Fig. 4d and Supplementary Fig. 18) because optical resonances improve in some spectral regions, but decrease in others, the absorbance.

We also calculated the internal quantum efficiency IQE using the measured EQE and simulated light absorption in the CQD active layer (Fig. 4f). Multibandgap CQD ensembles show enhanced EQE and IQE compared to pure CQD films, as transport of photogenerated charges takes place mainly through low defect-density, small-bandgap CQD paths. The enhanced EQE in multi-bandgap CQD ensembles shows not only the improved spectral range from the extended absorption, but also the enhanced transport, higher than pure CQD films, as was demonstrated by FET results.

We finally investigated the thickness-dependent performance of the pure and mixed CQD films (Supplementary Fig. 16) The optimal thickness for every device is found to be around $300 \mathrm{~nm}$, where $J_{\mathrm{SC}}$ decreases as the thickness increases due to resonant absorption as discussed above, which is in good agreement with the double pass absorption and simulation in Fig. 3 and Supplementary Fig. 10. For different inclusions of L, the $67 \%$ of L CQDs yields the highest $J_{\mathrm{SC}}$ of $3.7 \mathrm{~mA} \mathrm{~cm}^{-2}$ at a thickness of $300 \mathrm{~nm}$, whereas the 50 and $33 \%$ of L CQDs both yield the highest $J_{\mathrm{SC}}$ of $3.4 \mathrm{~cm}^{-2}$ when they are $320 \mathrm{~nm}$ thick.

\section{Discussion}

In this work, we report a strategy based on multi-bandgap CQD ensembles to achieve high open-circuit voltage, short-circuit current and PCE in cSi-filtered IR photovoltaics. We engineered the DOSs in this platform to improve quasi-Fermi level splitting and increase $V_{\text {OC. }}$. We further leveraged the optical properties of multi-bandgap CQD ensembles to achieve solar-matched IR light absorption, leading to high $J_{\mathrm{SC}}$ and a record cSi-filtered power conversion efficiency of $1 \%$, setting a record for silicon-filtered CQD PVs. This strategy, which allows decoupling of the traditional $V_{\mathrm{OC}}-J_{\mathrm{SC}}$ trade-off, has the potential to raise the IR PCE in the direction of the $6 \%$ theoretical limit with the improved light absorption properties of a mixture of CQD populations wellmatched to the solar spectrum.

\section{Methods}

Materials and characterization. The oleate-capped PbS CQDs and $\mathrm{ZnO}$ nanoparticles were synthesized following our previous reports ${ }^{24}$. Other chemicals were obtained from commercial suppliers and used as is. Optical absorption measurements were performed on a Lambda 950 UV-Vis-IR spectrometer.
QDs ligand exchange and solution preparation. The $\mathrm{PbI}_{2} / \mathrm{Pb}(\mathrm{SCN})_{2} / \mathrm{AA} \mathrm{DMF}$ solution ligand exchange is carried out in a test tube in air. Precursor solution $\left(\mathrm{PbI}_{2}\right.$ $0.1 \mathrm{M}, \mathrm{AA} 0.02 \mathrm{M}$ for $1150 \mathrm{~nm}$ CQDs, and $\mathrm{PbI}_{2} 0.1 \mathrm{M}$, BTA $0.04 \mathrm{M}$, and AA $0.06 \mathrm{M}$ for $1250 \mathrm{~nm}$ CQDs) is dissolved in DMF. $0.5 \mathrm{ml}$ of oleate-capped PbS CQDs octane solution $\left(50 \mathrm{mg} \mathrm{ml}^{-1}\right)$ was added to $5 \mathrm{ml}$ of precursor solution, followed by vigorously mixing for $2 \mathrm{~min}$ until the CQDs completely transferred to the DMF phase. The DMF phase was then washed three times with octane. Then $1150 \mathrm{~nm}$ CQDs precipitated during the exchange, while $1250 \mathrm{~nm}$ CQDs are stable in DMF and precipitated by adding $4 \mathrm{~mL}$ of acetone. The CQD precipitates were collected by centrifugation, followed by vacuum drying for $15 \mathrm{~min}$. The CQDs were redispersed in a mixture of BTA and DMF at a volume ratio of $8 / 2\left(250 \mathrm{mg} \mathrm{ml}^{-1}\right)$ for film by spin coating.

FET fabrication. Bottom-gate top-contact FET configuration is used as follows: $70 \mathrm{~nm}$ of titanium gate was thermally evaporated onto a glass substrate, followed by $15 \mathrm{~nm}$ of $\mathrm{ZrO}_{2}$ as a dielectric layer using atomic layer deposition (ALD). After $300^{\circ} \mathrm{C}$ baking for $1 \mathrm{~h}$, the pre-exchanged QDs dissolved in BTA/DMF were spincoated onto the substrate. Then $70 \mathrm{~nm}$ of Au source/drain electrodes were thermally deposited using an Angstrom Engineering Amod deposition system. Agilent $4155 \mathrm{c}$ semiconductor analyzer was used to characterize the FET devices.

CQD solar cell fabrication. $\mathrm{ZnO}$ layer was adopted as electron acceptor layer and formed on ITO-coated glass substrate by spin coating the $\mathrm{ZnO}$ nanoparticles solution at $3000 \mathrm{rpm}$ for $30 \mathrm{~s}$. Then PbS CQDs (pure CQDs or mixtures with different weight ratio), $250 \mathrm{mg} \mathrm{mL}^{-1}$ in BTA/DMF (8/2 volume ratio) solution, were spin cast on $\mathrm{ZnO}$ substrate at $2500 \mathrm{RPM}$ for $30 \mathrm{~s}$, followed by two layers of EDT-exchanged PbS CQDs as follows: 2 drops of oleic acid-capped PbS CQDs octane solution $\left(50 \mathrm{mg} \mathrm{mL}^{-1}\right)$ were spin coated at $2500 \mathrm{rpm}$ for $10 \mathrm{~s}$, followed by soaking in $0.01 \% \mathrm{EDT}$ in acetonitrile (ACN) solution for $30 \mathrm{~s}$ and washing with $\mathrm{ACN}$ for 3 times. For the top electrode, $120 \mathrm{~nm}$ of Au was deposited on EDT PbS CQD film to complete the device.

External and internal quantum efficiency. EQE and IQE spectra were acquired on a QuantX-300 quantum efficiency measurement system (Newport). Monochromated white light from a xenon lamp was mechanically chopped at a frequency of $25 \mathrm{~Hz}$. EQE spectra were acquired at zero electrical bias, whereas IQE spectra were calculated from an EQE spectra taken at a negative bias of $-2 \mathrm{~V}$ using the following formula: $\mathrm{IQE}=\mathrm{EQE}(0 \mathrm{~V}) / \mathrm{EQE}(-2 \mathrm{~V})^{34}$.

Current-voltage under simulated AM1.5. The current-voltage behavior under a simulated AM1.5 solar spectrum was acquired and corrected according to EQE spectra. Devices were kept in an inert $\mathrm{N}_{2}$ atmosphere. The input power density was adjusted to 1 Sun using a NIST-traceable calibrated reference cell (Newport 91150 V). To account for the spectral mismatch between the AM1.5 G reference spectrum and the spectrum of the lamp, a current density correction factor was used for each device, corresponding to the ratio of the value calculated from integrating the EQE spectrum and the value measured under illumination ${ }^{35}$. The lamp spectrum was measured using irradiance-calibrated spectrometers (USB2000 and NIR512, Ocean Optics) and is shown in Supplementary Fig. 17. The calculated spectral mismatch factors are shown in Supplementary Table 3.

Ultrafast transient absorption spectroscopy. A regeneratively amplified $\mathrm{Yb}$ : KGW laser (PHAROS, Light Conversion) laser was used to generate femtosecond pulses (250 fs FWHM) at $1030 \mathrm{~nm}$ as the fundamental beam with a $5 \mathrm{kHz}$ repetition rate. This fundamental beam was passed through a beam-splitter, where one arm was used to pump an optical parametric amplifier (ORPHEUS, Light Conversion) for the narrowband pump, and the other arm was focused into a sapphire crystal (Ultrafast Systems) in order to generate a NIR white-light continuum probe with a spectral window of $1050 \mathrm{~nm}$ to $1600 \mathrm{~nm}$. Both arms were directed into a commercial transient absorption spectrometer (Helios, Ultrafast Systems). The probe pulse was delayed relative to the pump pulse to provide a time window of up to $8 \mathrm{~ns}$. All measurements were performed using an average power of $100 \mu \mathrm{W}$ with a spot size of $0.40 \mu \mathrm{m}^{2}$, assuming a Gaussian beam profile.

\section{Data availability}

All relevant data are available from the authors upon request.

Received: 14 December 2017 Accepted: 23 August 2018

Published online: 01 October 2018

\section{References}

1. Technology Roadmap for Photovoltaic (ITRPV): 2015 Results Including Maturity Report (VDMA Photovoltaic Equipment, 2016). 
2. Richter, A., Hermle, M. \& Glunz, S. W. Reassessment of the limiting efficiency for crystalline silicon solar cells. IEEE J. Photovolt. 3, 1184-1191 (2013).

3. Sargent, E. H. Infrared photovoltaics made by solution processing. Nat. Photonics 3, 325-331 (2009).

4. Ip, A. H. et al. Infrared colloidal quantum dot photovoltaics via coupling enhancement and agglomeration suppression. ACS Nano 9, 8833-8842 (2015).

5. Yu, W. W., Wang, Y. A.. \& Peng, X. Formation and stability of size-, shape-, and structure-controlled CdTe nanocrystals: ligand effects on monomers and nanocrystals. Chem. Mater. 15, 4300-4308 (2003).

6. Yuan, M., Liu, M. \& Sargent, E. H. Colloidal quantum dot solids for solutionprocessed solar cells. Nat. Energy 1, 16016 (2016).

7. Lan, X. et al. Passivation using molecular halides increases quantum dot solar cell performance. Adv. Mater. 28, 299-304 (2016).

8. Chuang, C. H. M. et al. Open-circuit voltage deficit, radiative sub-bandgap states, and prospects in quantum dot solar cells. Nano Lett. 15, 3286-3294 (2015).

9. Liu, M. et al. Hybrid organic-inorganic inks flatten the energy landscape in colloidal quantum dot solids. Nat. Mater. 16, 258-263 (2016).

10. Kagan, C. R., Lifshitz, E., Sargent, E. H. \& Talapin, D. V. Building devices from colloidal quantum dots. Science 353, aac5523 (2016).

11. Cao, Y., Stavrinadis, A., Lasanta, T., So, D. \& Konstantatos, G. The role of surface passivation for efficient and photostable $\mathrm{PbS}$ quantum dot solar cells. Nat. Energy 1, 16035 (2016).

12. $\mathrm{Lu}, \mathrm{K}$. et al. High-efficiency $\mathrm{PbS}$ quantum-dot solar cells with greatly simplified fabrication processing via 'solvent-curing'. Adv. Mater. 1707572, 1-9 (2018).

13. Ouellette, O. et al. Optical resonance engineering for infrared colloidal quantum dot photovoltaics. ACS Energy Lett. 1, 852-857 (2016).

14. Kiani, A. et al. Single-step colloidal quantum dot films for infrared solar harvesting. Appl. Phys. Lett. 109, 183105 (2016).

15. Shockley, W. \& Queisser, H. J. Detailed balance limit of efficiency of p-n junction solar cells. J. Appl. Phys. 32, 510-519 (1961).

16. Rühle, S. Tabulated values of the Shockley-Queisser limit for single junction solar cells. Sol. Energy 130, 139-147 (2016).

17. Jo, J. W. et al. Enhanced open-circuit voltage in colloidal quantum dot photovoltaics via reactivity-controlled solution-phase ligand exchange. $A d v$. Mater. 1703627, 1-6 (2017).

18. Wang, H., Kubo, T., Nakazaki, J. \& Segawa, H. Solution-processed short-wave infrared $\mathrm{PbS}$ colloidal quantum Dot/ZnO nanowire solar cells giving high open-circuit voltage. ACS Energy Lett. 2, 2110-2117 (2017).

19. Carey, G. H., Levina, L., Comin, R., Voznyy, O. \& Sargent, E. H. Record charge carrier diffusion length in colloidal quantum dot solids via mutual dot-to-dot surface passivation. Adv. Mater. 27, 3325-3330 (2015).

20. Sun, Z., Sitbon, G., Pons, T., Bakulin, A. A. \& Chen, Z. Reduced carrier recombination in PbS - CuInS2 quantum dot solar cells. Sci. Rep. 5, 10626 (2015).

21. Fermi, E. Sulla quantizzazione del gas perfetto monoatomico. Rend. Lincei 3 145-149 (1927).

22. Ibach, H. \& Lüth, H. Solid-State Physics (Springer, 2009).

23. Guyot-Sionnest, P. Electrical transport in colloidal quantum dot films. J. Phys. Chem. Lett. 3, 1169-1175 (2012).

24. Felekidis, N., Wang, E. \& Kemerink, M. Open circuit voltage and efficiency in ternary organic photovoltaic blends. Energy Environ. Sci. 9, 257-266 (2016).

25. Garcia-Belmonte, G. \& Bisquert, J. Open-circuit voltage limit caused by recombination through tail states in bulk heterojunction polymer-fullerene solar cells. Appl. Phys. Lett. 96, 94-97 (2010).

26. Moreels, I. et al. Size-dependent optical properties of colloidal $\{\mathrm{PbS}\}$ quantum dots. ACS Nano 3, 3023-3030 (2009).

27. Würfel P., Würfel U. Physics of Solar Cells: From Basic Principles to Advanced Concepts 3rd edn (Wiley-VCH, Germany, 2016).

28. Sun, B. et al. Pseudohalide-exchanged quantum dot solids achieve record quantum efficiency in infrared photovoltaics. Adv. Mater. 1700749, 1-5 (2017).
29. Sandeep, C. S. S. et al. Epitaxially connected PbSe quantum-dot films: controlled neck formation and optoelectronic properties. ACS Nano $\mathbf{8}$, 11499-11511 (2014)

30. Balazs, D. M. et al. Stoichiometric control of the density of states in $\mathrm{PbS}$ colloidal quantum dot solids. Sci. Adv. 3, 1-8 (2017).

31. Oh, S. J. et al. Engineering the surface chemistry of lead chalcogenide nanocrystal solids to enhance carrier mobility and lifetime in optoelectronic devices. Chem. Commun. 53, 728-731 (2017)

32. Burkhard, G. F., Hoke, E. T. \& McGehee, M. D. Accounting for interference, scattering, and electrode absorption to make accurate internal quantum efficiency measurements in organic and other thin solar cells. Adv. Mater. 22, 3293-3297 (2010)

33. Orfanidis, S. J. Electromagnetic Waves and Antennas. (Rutgers University, U.S A., 2016).

34. Lin, Q., Armin, A., Nagiri, R. C. R., Burn, P. L. \& Meredith, P. Electro-optics of perovskite solar cells. Nat. Photonics 9, 106-112 (2015).

35. Shrotriya, V. et al. Accurate measurement and characterization of organic solar cells. Adv. Funct. Mater. 16, 2016-2023 (2006)

\section{Acknowledgements}

This work was supported by Ontario Research Fund-Research Excellence program (ORF7-Ministry of Research and Innovation, Ontario Research Fund-Research Excellence Round 7), and by the Natural Sciences and Engineering Research Council (NSERC) of Canada. M.I.S. acknowledges the support of the Banting Postdoctoral Fellowship Program, administered by the Government of Canada.

\section{Author contributions}

B.S., O.O., F.P.G.A., S.H., S.O.K. and E.H.S. designed and directed this study. B.S., Y.H.K. M.W., A.H.P., M.I.S., J.X., M.L., P.L., J.Z.F., J.W.J., H.T., F.T. and Z.H.L. carried out the synthesis, ligand exchange, characterizations, and solar-cell device fabrication. O.O., F.P. G.A. and O.V. performed the simulation studies. B.S., O.O., F.P.G.A., O.V. and E.H.S. wrote the manuscript.

\section{Additional information}

Supplementary Information accompanies this paper at https://doi.org/10.1038/s41467018-06342-7.

Competing interests: The authors declare no competing interests.

Reprints and permission information is available online at http://npg.nature.com/ reprintsandpermissions/

Publisher's note: Springer Nature remains neutral with regard to jurisdictional claims in published maps and institutional affiliations.

\begin{abstract}
Open Access This article is licensed under a Creative Commons Attribution 4.0 International License, which permits use, sharing, adaptation, distribution and reproduction in any medium or format, as long as you give appropriate credit to the original author(s) and the source, provide a link to the Creative Commons license, and indicate if changes were made. The images or other third party material in this article are included in the article's Creative Commons license, unless indicated otherwise in a credit line to the material. If material is not included in the article's Creative Commons license and your intended use is not permitted by statutory regulation or exceeds the permitted use, you will need to obtain permission directly from the copyright holder. To view a copy of this license, visit http://creativecommons.org/ licenses/by/4.0/.
\end{abstract}

(c) The Author(s) 2018 